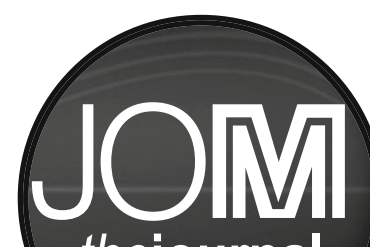
thejournal

\title{
2014 and 2015 editorial calendar
}

\section{September 2014}

Theme: Metals and Alloys: Part II

- Energy Savings in Mining and Metallurgical Industries

- Critical Metals Hydrometallurgy

- Modeling, Simulation and Development of Metallurgical Processes

- Refractory Metals and Alloys

- Low-Density Steels

Manuscripts Due: Deadline Passed

\section{October 2014}

Theme: Mechanical Properties

- Progress in High-Entropy Alloys

- Multi-Objective Optimization for Materials

- Multiscale Perspective of Interface-related Mechanics of Nanocomposites

- Deformation and Forming of Joined Materials

- Multi-scale Modeling: Concurrent and Hierarchical Methods

Manuscripts Due: Deadline Passed

\section{November 2014}

Theme: Energy and Environment

- Aluminum: Recycling and Environmental Issues

- Alloys and Compounds for Thermoelectric and Solar Cell Applications

- Progress with Lead-free Solders

- Critical Materials: Strategies for Achieving Sustainability

Manuscripts Due: Deadline Passed

\section{December 2014}

Theme: Materials for Extreme Environments

- High-Temperature Coatings for Environmental Protection

- Nuclear Applications of ODS and NFA Alloys

- Long-Term Durability of High-Temperature Alloys

- Long-Term Stability of High-Temperature Materials

- Radiation Effects in Oxide Ceramics and Novel LWR Fuels

Manuscripts Due: September 1, 2014

\section{January 2015}

Theme: Applying Materials Science and Engineering

- Scalable Nanomanufacturing

- Corrosion Fatigue

Manuscripts Due: September 15, 2014

February 2015

Theme: Upstream Materials: Extraction, Processing and Characterization

- Aluminum: Bauxite-Alumina-Carbon-Reduction

- Characterization of Advanced Mineral Materials

- Lithium Metal and Chemical Extraction and Processing

Manuscripts Due: October 15, 2014

\section{March 2015}

Theme: Additive Manufacturing

- Modeling of Microstructure Evolution during Additive Manufacturing

- Metal Powder for Additive Manufacturing (3D Printing)

- Progress in Additive Manufacturing

Manuscripts Due: November 15, 2014

\section{April 2015}

Theme: Biomaterials and Thin Films

- Nanomechanical Behavior of Biomaterials

- Surfaces and Biointerfaces

- Electronic, Biological, and Functional Thin Films

- Archaeomaterials

Manuscripts Due: December 15, 2014

\section{May 2015}

Theme: Metallurgy and Processing

- Aluminum: Shaping and Forming

- Friction Stir Welding and Processing

- Metallurgy: Energy and Environmental Issues

Manuscripts Due: January 15, 2015

June 2015

\section{Theme: Metals and Alloys}

- Beta-Titanium Alloys

- Permanent Magnets beyond Nd-Dy-Fe-B

- Critical Materials: Strategies for Achieving Sustainability

- TMS2015 Bladesmithing Project

Manuscripts Due: February 15, 2015 


\section{4 and 2015 advisors and committees}

Amit Pandey

Advanced Characterization, Testing, and Simulation Committee

Michael Gao and Sinn-wen Chen Alloy Phases Committee

Alton Tabereaux Aluminum Committee

Candan Tamerler-Behar

Biomaterials Committee

Michael J. Demkowicz

Chemistry and Physics of Materials Committee

Muralidharan Paramsothy Composite Materials Committee

Bala Radhakrishnan Computational Materials Science and

Engineering Committee

Vilupanur A. Ravi (2014)

Kinga Unocic (2015)

Corrosion \& Environmental Effects Committee

Babak Arfaei

Electronic Packaging \&

Interconnection Materials Committee

Cong Wang

Energy Committee

Xiaochuan Lu

Energy Conversion and Storage

Committee
Chantal Sudbrack

High Temperature Alloys Committee

Harald Oosterhof

Hydrometallurgy \& Electrometallurgy

Committee

Nitin Chopra

ICME Committee

James Saal

Magnesium Committee

Satoshi Hirosawa

Magnetic Materials Committee

Bowen Li

Materials Characterization

Committee

Marian S. Kennedy

Mechanical Behavior of Materials

Committee

Terry Xu and Jung-Kun Lee

Nanomaterials Committee

Jian Wang (2014-2015)

Xiaodong Li (2015)

Peter Hosemann (2015)

Nanomechanical Materials Behavior Committee

Raul Rebak (2014)

Ramprashad Prabhakaran (2015)

Nuclear Materials Committee
Amy Clarke

Phase Transformations Committee

Ma Qian

Powder Materials Committee

Antoine Allanore

Process Technology \& Modeling

Committee

Philip J. Mackey (2014)

Zhiwei Peng (2014-2015)

Dean Gregurek (2014-2015)

Pyrometallurgy Committee

Gabrielle Gaustad

Recycling \& Environmental

Technologies Committee

Todd Leonhardt

Refractory Metals Committee

John Carsley

Shaping \& Forming Committee

André Phillion (2014)

Mohaen Asle Zaeem (2015)

Solidification Committee

Sandip Harimkar and

Srinivasa Bakshi

Surface Engineering Committee

Roger Narayan

Thin Films \& Interfaces

Committee

William Joost

Titanium Committee

Anyone wishing to publish in JOM should follow the guidelines established in the JOM Instructions for Authors, which features detailed information on communication, manuscript preparation, and publication procedures. The Instructions for Authors are available on the JOM website at www.jom.tms.org.

For More Information Contact:

Telephone: (724) 776-9000 ext. 228

e-mail: jom@tms.org 